\title{
The Functional Anatomy of Sound Intensity Discrimination
}

\author{
Pascal Belin, ${ }^{1}$ Stephen McAdams,, ${ }^{2,3}$ Bennet Smith, ${ }^{2}$ Sophie Savel, ${ }^{2,3}$ Lionel Thivard, ${ }^{1}$ Séverine Samson, ${ }^{4}$ \\ Yves Samson ${ }^{1,5}$
}

${ }^{1}$ Groupe de Neurologie, Service Hospitalier Frédéric Joliot, DRM-CEA, F-91406 Orsay cedex, France, 2Institut de Recherche et de Coordination Acoustique/Musique, F-75004 Paris, France, ${ }^{3}$ Laboratoire de Psychologie Expérimentale (Centre National de la Recherche Scientifique), Université René Descartes, Équipe de Neuropsychologie et Langage, F75006 Paris, France, ${ }^{4}$ Université Charles de Gaulle Lille III, BP 149, F-59653 Villeneuve d'Asq cedex, France, and ${ }^{5}$ Service des Urgences Cérébro-Vasculaires, Hôpital de la Salpêtrière, F-75651 Paris cedex 13, France

The human neuroanatomical substrate of sound intensity discrimination was investigated by combining psychoacoustics and functional neuroimaging. Seven normal subjects were trained to detect deviant sounds presented with a slightly higher intensity than a standard harmonic sound, using a Go/No Go paradigm. Individual psychometric curves were carefully assessed using a three-step psychoacoustic procedure. Subjects were scanned while passively listening to the standard sound and while discriminating changes in sound intensity at four different performance levels $\left(d^{\prime}=1.5,2.5,3.5\right.$, and 4.5). Analysis of regional cerebral blood flow data outlined activation, during the discrimination conditions, of a right hemispheric frontoparietal network already reported in other studies of selective or sustained attention to sensory input, and in which activity appeared inversely proportional to intensity discriminability. Conversely, a right posterior temporal region included in secondary auditory cortex was activated during discrimination of sound intensity independently of performance level. These findings suggest that discrimination of sound intensity involves two different cortical networks: a supramodal right frontoparietal network responsible for allocation of sensory attentional resources, and a region of secondary auditory cortex specifically involved in sensory computation of sound intensity differences.

Key words: audition; attention; intensity discrimination; functional neuroimaging; psychoacoustics; human; performance level
Most functional neuroimaging studies of the auditory system so far have focused on complex aspects of auditory function [e.g., functional lateralization and phonological processing (Knopman et al., 1982; Démonet et al., 1992, 1994; Zatorre et al., 1992, 1996; Binder et al., 1994; Fiez et al., 1995; O'Leary et al., 1995; Johnsrude et al., 1997; Platel et al., 1997; Wessinger et al., 1997; Belin et al., 1998)]. Few authors have used functional neuroimaging techniques to study the perception of what is probably the most basic feature of auditory signals and considerably affects their neural processing, i.e., sound intensity. At a cellular level, intensity of auditory stimulation has been shown to dramatically modulate the firing rate of most neurons in the auditory cortex within a certain range (Brugge and Reale, 1985; Ehret and Merzenich, 1988). Furthermore, recent electrophysiological studies in animals have identified regions of secondary cortex in which the nonmonotonic neural responses to intensity changes suggest a possible role for intensity discrimination (Heil and Irvine, 1998). At a more general level, sound intensity conveys crucial information about the vibratory phenomenon that produces the sound, i.e., its strength and its distance from the listener. Variations in sound intensity are important in many aspects of auditory cogni-

\footnotetext{
Received Feb. 17, 1998; revised May 29, 1998; accepted June 1, 1998.

This work was supported by Fondation France-Télécom and Groupement d' Intérêt Scientifique-Sciences de la Cognition. We thank the staff members of the Institut de Recherche et de Coordination Acoustique/Musique and the Orsay Brain Imaging Center for technical assistance. We are grateful to R. J. Zatorre for his useful comments on this manuscript.

Correspondence should be addressed to Y. Samson, Service des Urgences Cérébro-Vasculaires, Hôpital de la Salpêtrière, 47 boulevard de l'Hôpital, F-75651 Paris cedex 13, France.

Copyright (ㄷ) 1998 Society for Neuroscience $\quad 0270-6474 / 98 / 186388-07 \$ 05.00 / 0$
}

tion, such as estimating the radial movement of a source or perceiving subtle meanings in a verbal message conveyed by speech prosody (Monrad-Krohn, 1963; Joanette et al., 1994). These considerations, as well as electrophysiological data in humans (Näätänen, 1990; Giard et al., 1995), strongly suggest that the cerebral cortex plays a critical role in perceiving small changes in sound intensity, yet little is known about the localization of the cortical structures involved in such processing.

In the present study, positron emission tomography (PET) and psychoacoustics were combined to identify, in human subjects, the cortical network involved in detection of changes in sound intensity. Normal volunteers were trained to discriminate small intensity differences in a Go/No Go paradigm. To ensure that such discrimination would be performed at equivalent levels of performance for each subject, individual psychometric curves were carefully assessed using a three-step psychoacoustic strategy. Measures of regional cerebral blood flow ( $\mathrm{rCBF}$ ) were then performed while subjects passively listened to standard sounds (baseline condition) and while they detected deviant sounds of slightly higher intensity among these standard sounds (discrimination conditions). Four different discrimination levels were used, corresponding to equivalent levels of performance across subjects as assessed by the $d^{\prime}$ index $\left(d^{\prime}=4.5,3.5,2.5\right.$, and 1.5 , with higher $d^{\prime}$ indicating greater sensitivity and thus greater ease of discrimination). Functional images were analyzed with statistical parametric mapping, using categorical (intensity discrimination vs baseline), parametric ( $\mathrm{rCBF}-d^{\prime}$ index correlation), and multivariate (principal-component analysis) designs. We predicted that certain cortical regions would be specifically activated by discrimination of auditory intensity differences, and we were 
especially interested in the relation between rCBF (indirect index of neuronal activity) and discrimination performance in these areas.

\section{MATERIALS AND METHODS}

\section{Subjects}

Seven normal male volunteers (age 19-28 years) gave written informed consent. They had no history of neurological or psychiatric disorders and self-reported their audition as normal. The study was approved by the ethics committee of the La Salpétrière Hospital.

\section{Auditory stimuli}

Auditory stimuli were synthesized at a sampling rate of $44.1 \mathrm{kHz}$ using the Institut de Recherche et de Coordination Acoustique/Musique Musical Workstation (ISPW digital signal processing card and a NeXT computer). The stimuli were harmonic complexes with 20 harmonics and a fundamental frequency of $200 \mathrm{~Hz}$. The relative amplitudes of the harmonics were determined by a $1 / n$ spectral envelope, where $n$ is the harmonic rank ( $-3 \mathrm{~dB} /$ octave slope in the power spectrum). Each stimulus had a duration of $300 \mathrm{msec}$, including $80 \mathrm{msec}$ linear rise and decay ramps in the amplitude envelope. All standard sounds were presented binaurally over earphones at a level of $75 \mathrm{~dB}$ sound pressure level (SPL) as measured with a Bruel and Kjaer 2209 sound level meter.

\section{Psychoacoustic measures}

The main task used in the imaging studies was Go/No Go. In a series of events of which the majority $(75 \%)$ have the reference level and the minority $(25 \%)$ have a level that is greater by some chosen amount, the subject must decide whether each one is the reference value (in which case no action is taken, No Go) or a deviant value, which is always superior to the reference value (in which case the change is noted mentally in the imaging task and a button is pushed in the psychoacoustic task, Go). Ideally it should be possible to establish performance levels for this kind of task with varying level differences. However, this task has not been studied much in human psychoacoustics, and the data therefore cannot be compared with the literature. The psychoacoustic measures were thus made using both a classic same/different task [same/different (Phase 2)] with constant stimuli as well as the Go/No Go task (Phase 3).

Phase 1: Preliminary measure of high and low thresholds by an adaptive method. To reduce experimentation time, a first rough measure of each subject's sensitivity to level change was made using an $N$-down, 1 -up adaptive procedure (Levitt, 1971), which converges on a performance level that depends on $N$. $N$ consecutive correct responses result in a decrease in level difference, and one incorrect response results in an increase. In our case, $N$ was 3 [79.4\%, low threshold (TL)] and $8(91.7 \%$, high threshold $(\mathrm{TH})]$. The levels at which the adaptive trajectory changes direction were recorded, and the last 8 of 12 were averaged to estimate TL, and the last four of six were averaged for TH. Six estimates were obtained for each threshold. From the mean level differences obtained at each threshold for each subject, the values used in Phase 2 were determined. If half the level difference between TL and TH is denoted $d$, the tested levels included TL $-d$, TL, TL $+d, \mathrm{TH}, \mathrm{TH}+d$.

Phase 2: Psychometric functions determined with the method of constant stimuli. For each of the five level differences obtained in Phase 1, a block of 200 trials was constructed. Each trial was composed of two sounds presented sequentially. Four combinations are possible: two reference stimuli (same), two test stimuli (same), and one of each in the two orders (different). There were 50 repetitions of each trial type in the block, presented in random order. After hearing the two sounds, the subject indicated whether the stimuli were the same or different. The hit rate was computed on "different" trials from the percentage of correct responses. The false alarm rate was computed on "same" trials from the percentage of incorrect responses. According to signal detection theory (Green and Swets, 1974), the discrimination rate expressed as percentage of correct detection of intensity change is influenced both by the subject's perceptual sensitivity and by his or her judgment strategy. Because the interest of this study was to determine neural correlates of sensitivity to intensity change, the sensitivity $\left(d^{\prime}\right)$ was estimated from hit and false alarm rates (Macmillan and Creelman, 1990). This $d^{\prime}$ value is considered to estimate true sensitivity to intensity difference with biases attributable to response strategy having been factored out. This procedure was repeated for each level difference in a random order for each subject. From the $d^{\prime}$ values estimated for each level difference, a psychometric function was determined from a linear regression of those $d^{\prime}$ values onto level differences.
The highest $d^{\prime}$ value was at times removed from the fitting procedure if the curve clearly asymptoted at maximum performance level.

Phase 3: Psychometric functions determined with the Go/No Go method. On the basis of the previously determined psychometric function, five new level differences were chosen for the Go/No Go procedure corresponding to performances in Phase 2 equivalent to $d^{\prime}$ values of 1,2 , 3,4 , and 5. Each difference was presented in a separate block of trials. A block lasted $\sim 2 \mathrm{~min}$, as in the imaging experiment. During this time 200 events were presented, of which $75 \%$ had the reference level and $25 \%$ the (higher) test level. The subject listened continuously and pressed a button as soon as a test event occurred. The events were presented at a rate of one per second ( $300 \mathrm{msec}$ stimulus, $700 \mathrm{msec}$ silence). If the subject pressed the button during the $1 \mathrm{sec}$ temporal window corresponding to the test signal, it was scored as a hit. If the button press occurred outside of this window, it was scored as a false alarm. The $d^{\prime}$ values were estimated from hits and false alarm rates (Macmillan and Creelman, 1990). The procedure was repeated for each of the five level differences in a different random order for each subject. From the $d^{\prime}$ values for each of the five level differences, a linear psychometric curve was determined as in Phase 2, and level differences corresponding to $d^{\prime}$ values of 1.5, 2.5, 3.5 , and 4.5 were chosen for the imaging studies for each subject.

\section{Functional neuroimaging}

Imaging. Relative $\mathrm{rCBF}$ was determined from the distribution of radioactivity after bolus intravenous injections of $\mathrm{H}_{2}{ }^{15} \mathrm{O}$ (Fox et al., 1984), measured with an ECAT-HR + PET camera (Siemens AG, Erlangen, Germany). Subjects received $12 \mathrm{H}_{2}{ }^{15} \mathrm{O}$ injections (9 $\mathrm{mCi}$ per injection) corresponding to $12 \mathrm{rCBF}$ measurements, performed at $10 \mathrm{~min}$ intervals. Attenuation-corrected data were reconstructed into 632.25 -mm-thick axial slices, with a resulting resolution of $4.5 \mathrm{~mm}$ full-width at halfmaximum after reconstruction (Bendriem et al., 1996).

Tasks. Four scans were acquired during a baseline condition, and eight scans were acquired during detection of intensity changes performed at four different levels of discriminability (two scans per level), in a counterbalanced order. The baseline condition consisted of passively listening to standard sounds, presented binaurally through Sony MDR-V600 headphones at a $75 \mathrm{~dB}$ SPL, with a 1 sec inter-onset interval. Subjects were informed that all sounds were identical and were instructed to listen carefully to the sounds. During the intensity discrimination conditions, subjects were instructed to mentally detect sounds of higher intensity (deviants), which were intermingled with the standard sounds of the baseline condition with a $25 \%$ probability of occurrence. The deviant sounds were identical to the standard sounds in all respects but intensity, which was slightly greater in the deviants. During a given intensity discrimination condition, all deviants were identical, with an intensity corresponding to a given performance level $\left(d^{\prime}=1.5,2.5,3.5\right.$, or 4.5$)$ for the scanned subject. To avoid possible contamination of the activation pattern by motor-specific activity, no overt response was required from the subjects. However, subjective intensity discriminability, as indicated by subjects after each scan, corresponded to the individual's objective $d^{\prime}$ value, thus confirming that they were performing the discrimination task as during the psychophysical sessions.

Data analysis. Statistical parametric mapping (SPM96) software was used for image realignment, transformation into standard stereotactic anatomical space (Talairach and Tournoux, 1988), smoothing, and statistical analysis (Friston et al., 1995a,b). State-dependent differences in global flow were covaried out using proportional scaling. Comparisons across conditions were made using the $t$ statistic subsequently transformed into the normally distributed $Z$ statistic $[\operatorname{SPM}(Z)]$. A categorical approach was first used to determine cerebral regions in which $\mathrm{rCBF}$ changed significantly in the seven subjects between baseline and the pooled discrimination conditions. A parametric approach was then used to determine regions in which $\mathrm{rCBF}$ covaried significantly in a linear way with level of performance $\left(d^{\prime}\right)$ during the intensity discrimination conditions. A principal components analysis was also performed on the dataset to determine a priori the composition of the activation/deactivation pattern (eigenimage) that best described the experimental dataset.

\section{RESULTS}

\section{Intensity discrimination}

The mean thresholds from all three phases of the psychoacoustic testing and psychometric functions for a typical subject (subject 2) are shown in Figure 1. Performance was highly variable across 


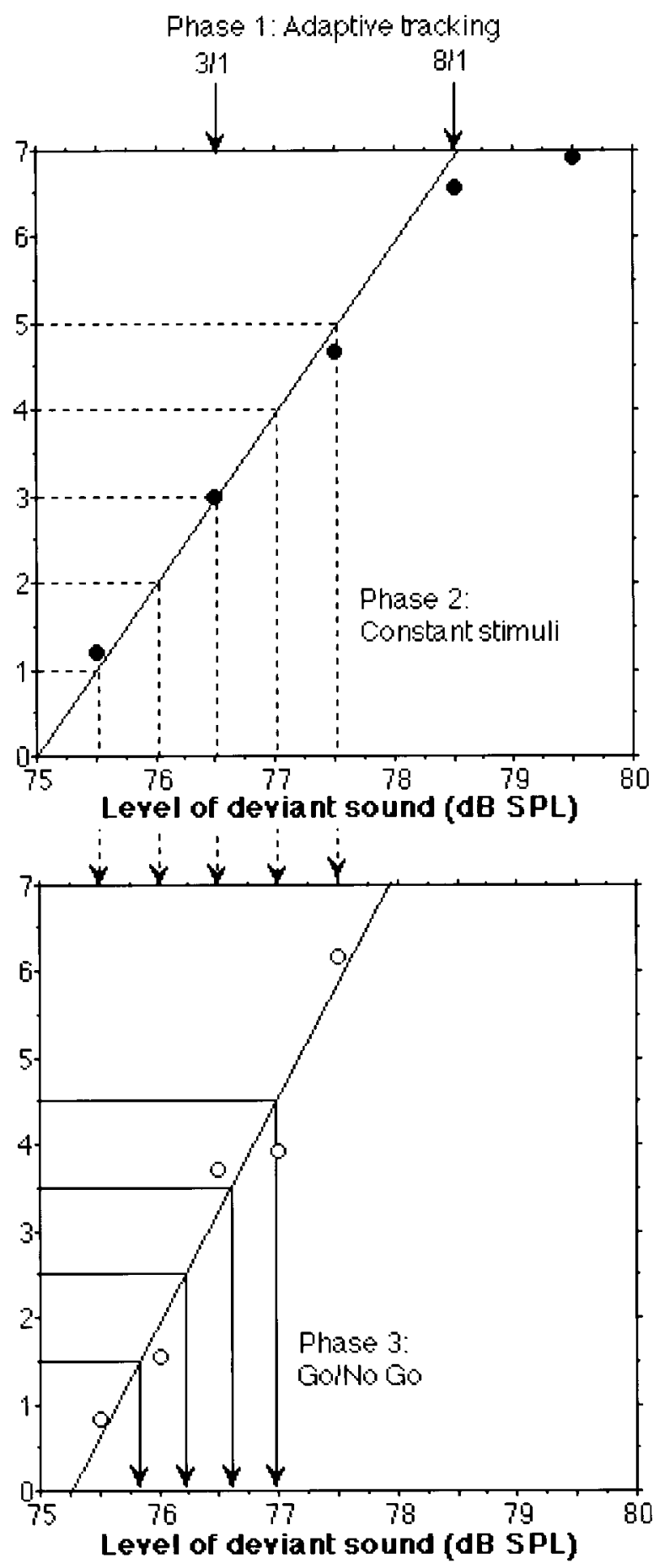

Figure 1. Psychophysical data for Subject 2. The two estimated thresholds from the three down/one up and eight down/one up adaptive procedures (Phase 1: Adaptive tracking, top panel) are shown with downward arrows. From these two values, the five stimulus levels for the constant stimuli procedure (Phase 2: Constant stimuli, top panel) were derived. A linear psychometric function (solid line) was fitted to the first four of the five data points obtained from this procedure $(\bullet)$. From this function, the five stimulus levels for the Go/No Go procedure (Phase 3: Go/No Go) were determined. A linear psychometric function (solid line) was then fitted to the five data points represented as open circles in the lower panel. Finally, the four stimulus levels used in the imaging study were derived from this latter function as indicated by the downward arrows in the bottom panel. subjects, resulting in varying physical differences (in decibels) for each $d^{\prime}$ value (Table 1 ). The $d^{\prime}$ values increase systematically and nearly linearly with increase in the level difference. The mean level difference discriminated by subjects varies from $1.1 \mathrm{~dB}$ at $d^{\prime}$ $=1.5$ to $2.7 \mathrm{~dB}$ at $d^{\prime}=4.5$. The inter-subject variability of the data is also greater for the higher levels of $d^{\prime}$, i.e., for more easily discriminated intensity differences (SDs of $0.63,0.79,0.96$, and $1.08 \mathrm{~dB}$ for $d^{\prime}$ values of $1.5,2.5,3.5$, and 4.5, respectively), indicating a divergence of the subjects' performance as a function of level difference. The relation between level and $d^{\prime}$ for each subject is linear, and the regression coefficients vary from 0.8 to nearly 1.0 (mean $=0.91)$. The pattern shown in Figure 1 is also similar for all subjects, i.e., the slope of the psychometric function for the constant-stimuli procedure is less than that for the $\mathrm{Go} / \mathrm{No}$ Go procedure, most likely indicating improvement in sensitivity over the course of the experiment. Despite the variability across subjects in terms of the physical difference at each sensitivity level, the use of such psychoacoustic methods ensures that the PET scan measures activation for four fixed levels of sensitivity in auditory discrimination that are similar across subjects.

\section{rCBF variations with detection of intensity changes}

When compared with the baseline, averaged detection conditions with similar weight for all four levels of performance yielded significant $\mathrm{rCBF}$ increases ( $p<0.05$ corrected for multiple nonindependent comparisons) located exclusively in the right cerebral hemisphere and in the contralateral left cerebellar hemisphere (Table 2, Fig. 2). Regions of maximal rCBF change were centered in the posterior part of the right superior temporal gyrus, caudally to Heschl's gyri [Brodmann Area (BA) 22/42], and in the right inferior frontal gyrus (BA 45). The latter region of significant $\mathrm{rCBF}$ increase extended posteriorly and superiorly along the precentral sulcus (BA 6), including two other maxima situated close to the anatomical location of the frontal eye field (Paus, 1996). A region in the right superior parietal cortex (dorsal part of the inferior parietal lobule, BA 40) was also significantly activated by the intensity-change detection task, as well as a region of the left dorsolateral cerebellar hemisphere. Figure 2 shows the anatomical location of these maxima on a right hemisphere surface rendering and the mean relative $\mathrm{rCBF}$ values corresponding to the baseline condition and to the four levels of intensity-change detection for each one of the four right hemisphere maxima. Note that for the parietal and frontal foci, a clear progression in mean rCBF value can be observed as $d^{\prime}$ decreases. On the contrary, $\mathrm{rCBF}$ in the posterior temporal focus appears largely independent of $d^{\prime}$.

Comparison of the averaged intensity discrimination conditions to the baseline also yielded regions of significant $\mathrm{rCBF}$ decreases $(p<0.05$ corrected $)$, all situated in the left cerebral hemisphere. These were located in the left inferior posterior parietal lobe (BA 39), in the left superior frontal gyrus (BA 8), and in the left inferior temporal pole (BA 20/38).

\section{rCBF: performance correlation}

A parametric approach was used to determine cerebral regions in which normalized activity was linearly related with equivalent performance level - as measured by $d^{\prime}$ value-during detection of intensity changes. A single region, located in right parietal cortex (BA 39,40), showed significant ( $p<0.01$ uncorrected) negative correlation between $\mathrm{rCBF}$ and $d^{\prime}$ value: decreasing discriminability (decreasing $d^{\prime}$ value) corresponded to linearly increasing mean rCBF value (Table 3 ). This region contained two 
Table 1. Stimulus levels (dB SPL) corresponding to the deviant sounds for detection of intensity changes at each performance level for each subject

\begin{tabular}{|c|c|c|c|c|c|c|c|c|c|}
\hline \multirow[b]{2}{*}{$d^{\prime}$} & \multicolumn{7}{|c|}{ Subject } & \multirow[b]{2}{*}{ Mean } & \multirow[b]{2}{*}{ SD } \\
\hline & No. 1 & No. 2 & No. 3 & No. 4 & No. 5 & No. 6 & No. 7 & & \\
\hline 1.5 & 77.4 & 75.8 & 75.9 & 75.9 & 76.2 & 75.6 & 75.6 & 76.1 & 0.63 \\
\hline 2.5 & 78.3 & 76.2 & 76.4 & 76.6 & 76.7 & 76.0 & 76.1 & 76.6 & 0.79 \\
\hline 3.5 & 79.2 & 76.6 & 76.8 & 77.3 & 77.1 & 76.5 & 76.5 & 77.1 & 0.96 \\
\hline 4.5 & 80.0 & 77.0 & 77.3 & 78.0 & 77.6 & 77.0 & 77.0 & 77.7 & 1.08 \\
\hline
\end{tabular}

Standard sounds were presented at $75 \mathrm{~dB}$ SPL.

Table 2. Brain activity associated with sound intensity discrimination compared with the baseline

\begin{tabular}{|c|c|c|c|c|c|}
\hline & $x$ & $y$ & $z$ & $Z$ value & Size \\
\hline \multicolumn{6}{|l|}{$\mathrm{rCBF}$ increases } \\
\hline Right posterior superior temporal gyrus (22/42) & 56 & -34 & 6 & 5.32 & 271 \\
\hline Right dorsal inferior parietal lobule (40) & 44 & -48 & 50 & 5.24 & 411 \\
\hline Right precentral sulcus (6) & 48 & 0 & 48 & 5.12 & 1806 \\
\hline Right inferior frontal operculum (45) & 42 & 28 & 4 & 5.06 & \\
\hline Right precentral sulcus (6) & 36 & 0 & 42 & 4.91 & \\
\hline Left dorsolateral cerebellar hemisphere & -38 & -62 & -26 & 4.51 & 151 \\
\hline \multicolumn{6}{|l|}{ rCBF decreases } \\
\hline Left posterior inferior parietal lobule (39) & -48 & -62 & 24 & 4.97 & 223 \\
\hline Left superior frontal gyrus (8) & -18 & 28 & 46 & 4.58 & 311 \\
\hline Left superior frontal gyrus (8) & -36 & 14 & 52 & 3.98 & \\
\hline
\end{tabular}

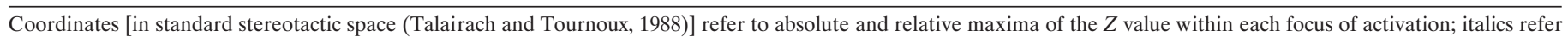

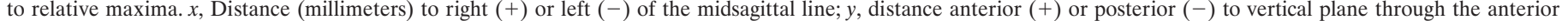

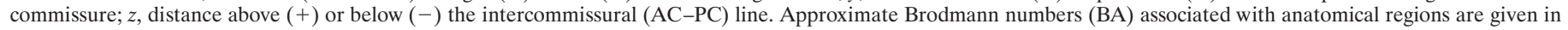

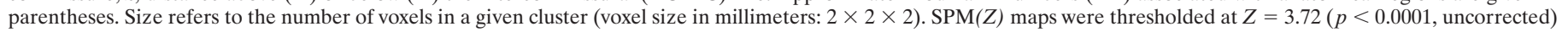
and then corrected for multiple nonindependent comparisons at $p<0.05$.

maxima, situated $\sim 2 \mathrm{~cm}$ ventrally and $1 \mathrm{~cm}$ caudally to the parietal activation peak obtained in the factorial analysis. No region showed significant $\mathrm{rCBF}$ increase with increasing discriminability (positive correlation with $d^{\prime}$ value). Importantly, no significant correlation subsisted, either positive or negative, when individual decibel values corresponding to each performance level were used instead of the common $d^{\prime}$ index.

\section{Eigenimage}

Additional insight was obtained by decomposing the dataset into principal components (eigenimages, or eigenvectors of the variance-covariance matrix). The first eigenimage alone explained $56.4 \%$ of the variance (Fig. 3). This eigenimage corresponded approximately to an activation/deactivation pattern obtained by contrasting the intensity discrimination conditions with the baseline conditions (Fig. 3, right). This result is very much similar to the one obtained with the regular factorial analysis, when identical weights were arbitrarily given for the different detection conditions. Maxima of rCBF increase were located in the right parietal and frontal lobe, as well as in the left cerebellar hemisphere. However, in striking contrast to the categorical activation/deactivation pattern, the previously maximally activated posterior temporal region completely disappears here. Concerning the composition of this eigenimage in terms of experimental conditions, a clear increase in the weights of the discrimination conditions along with decreasing discriminability (decreasing $d^{\prime}$ values) can be observed (Fig. 3).

\section{DISCUSSION}

\section{Right hemispheric frontoparietal network}

The right hemispheric frontoparietal activation pattern obtained during intensity discrimination is very consistent with those observed in previous neuroimaging studies involving sustained or selective attention to sensory input (Pardo et al., 1991; Gitelman et al., 1996; Paus et al., 1997). In the Pardo et al. (1991) study, subjects were instructed to attend to slight changes in visual or somatosensory stimulations. When compared with a reference resting state, the conditions of sustained attention to sensory stimuli-either visual or somatosensory-yielded consistent activation in the right parietal lobe [mean location: $x=39, y=-43$, $z=45$ in the Talairach atlas (Talairach and Tournoux, 1988)] and in the right frontal region extending from a superior precentral region (mean: $x=30, y=15, z=40$ ) to the inferior frontal gyrus $(x=39, y=9, z=9)$ (Pardo et al., 1991). In the Gitelman et al. (1996) study, spatial attention was examined in normal subjects by comparing active exploratory to passive hand movements. In addition to an anterior cingulate activation focus, the authors observed the characteristic right hemispheric frontoparietal network, with activation in BA 40 (mean: $x=35, y=-43, z=47$ ) and in the right prefrontal region (BA 6) close to the frontal eye field (mean: $x=46, y=9, z=32$ ) (Gitelman et al., 1996). In the Paus et al. (1997) study, subjects were scanned every $10 \mathrm{~min}$ during a 60 -min-long vigilance task that consisted of detecting decreases in intensity in auditory stimuli. Regions that showed the most significant $\mathrm{rCBF}$ decrease with time-interpreted as being 


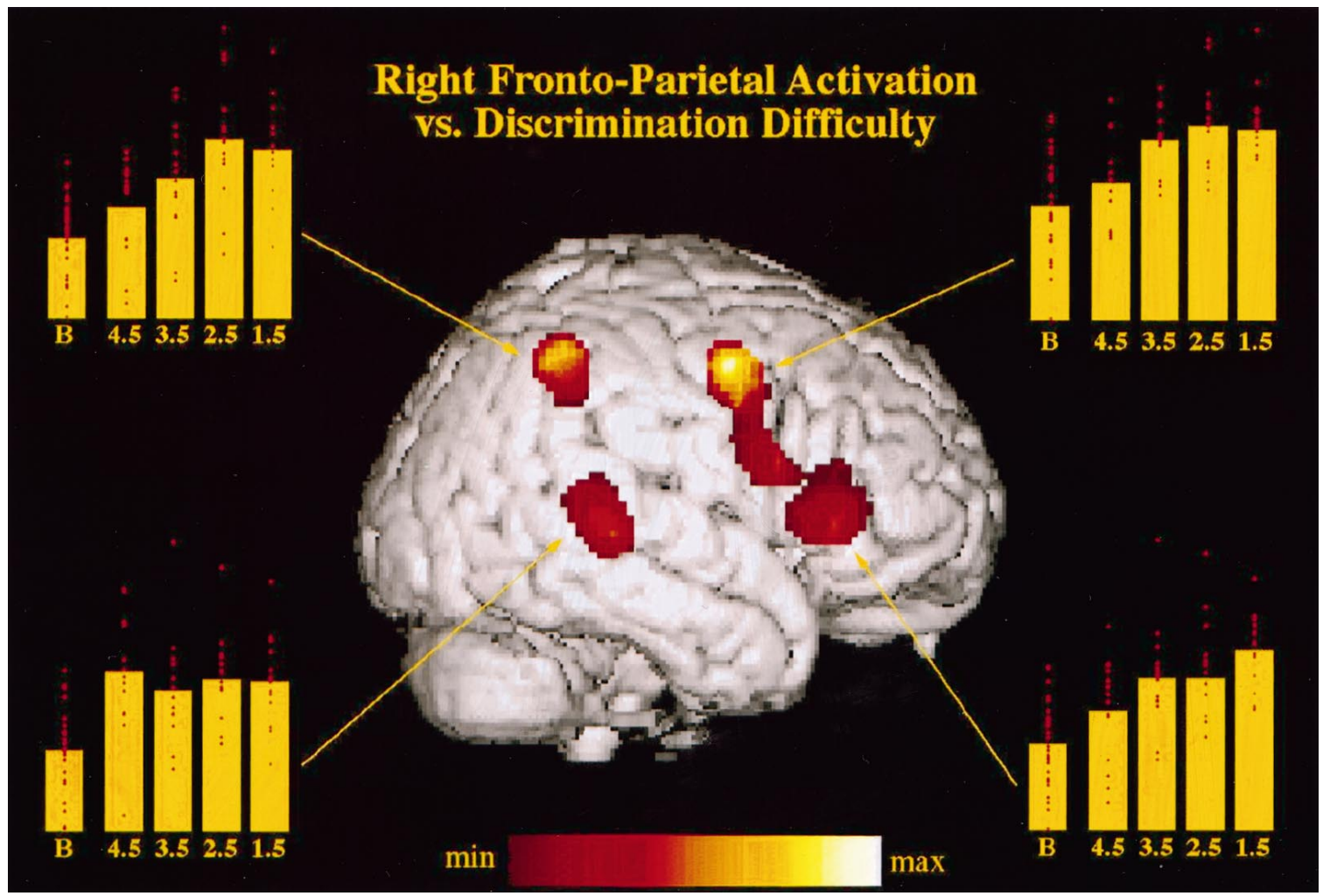

Figure 2. Surface rendering, on a T1 image of a right hemisphere, of the four cortical regions significantly activated during intensity discrimination (all performance levels pooled) as compared with the passive baseline. Yellow diagrams represent, for each of these regions, the mean (bar) and individual (red dots) $\mathrm{rCBF}$ values corresponding to the baseline $(B)$ and each of the four intensity discriminations $\left(d^{\prime}=1.5,2.5,3.5,4.5\right)$, in arbitrary units. Decreasing discriminability leads to increased activation in the frontoparietal network, but not in the posterior temporal region.

\begin{tabular}{|c|c|c|c|c|c|}
\hline & $x$ & $y$ & $z$ & $Z$ value & Size \\
\hline \multicolumn{6}{|c|}{ Negative rCBF- $d^{\prime}$ correlation } \\
\hline Right parietal (39/40) & 44 & -52 & 32 & 4.52 & 528 \\
\hline Right parietal (40/7) & 44 & -58 & 48 & 3.91 & \\
\hline
\end{tabular}

See Table 2 footnote. $\operatorname{SPM}(Z)$ maps were thresholded at $Z=2.33(p<0.01$ uncorrected).

part of the attentional network involved in sustained attention to the auditory stimuli-were located in the right inferior frontal gyrus (BA 45; maxi: $x=46, y=20, z=-2$ ) and in the right inferior parietal lobule (BA 40; maxi: $x=63, y=-35, z=36$ ) (Paus et al., 1997). These neuroimaging results, obtained for different sensory modalities, thus outline activation of a right hemispheric frontoparietal network that is very similar to the one observed in the present study for auditory stimuli presented in binaural conditions. This right frontoparietal network appears crucial to sustained attention to sensory input, as already suggested by animal and lesion data (Mesulam, 1981; Woods and Knight, 1986; Wilkins et al., 1987).

An important feature of our results is to show that activity of this right hemispheric frontoparietal network is modulated by the required attentional resource. It is very clear in our data that relative $\mathrm{rCBF}$ in this network increases with decreasing intensity discriminability (decreasing $d^{\prime}$ ). Figure 2 shows that in the parietal, superior prefrontal, and inferior prefrontal regions recruited by the intensity discrimination task, mean $\mathrm{rCBF}$ is in inverse relation to performance level $\left(d^{\prime}\right)$. The first eigenimage confirms this point (Fig. 3) by showing that the detection task contributes more and more to the activation of the frontoparietal network in the right hemisphere as discriminability decreases (increasing weights with decreasing $d^{\prime}$ ). Such a relation could be interpreted as underlying the increased attentional resources required to perform the discrimination task as discriminability decreases. Alternatively, it could also be related to the change in response criteria resulting in an increase in false alarm rate.

\section{Right posterior temporal region}

In contrast to activation of this general frontoparietal network is the activation of a region of secondary auditory cortex in the right posterior temporal gyrus (BA 42/22) that was observed during the detection tasks. This region, which corresponded to the greatest activation in the baseline/detection comparison, is probably selectively involved in detection of intensity changes for the following reasons. First, this posterior region was not found to be activated in neuroimaging studies that focused on other sensory modalities (Pardo et al., 1991; Gitelman et al., 1996), whereas a similar region of the right posterior superior temporal gyrus (maxi: $x=59, y=-21, z=-9$ ) was also found in the Paus et al. (1997) study for detection of intensity changes. Second, such activation is consistent with previous data from patients having undergone unilateral excision of the temporal lobe. When Milner et al. (1962) administered the Seashore Musical Aptitude battery to patients before and after surgical removal of the temporal lobe for relief of intractable epilepsy, performance in intensity discrimination was significantly poorer after removal only in the group of patients with excision within the right temporal lobe. 


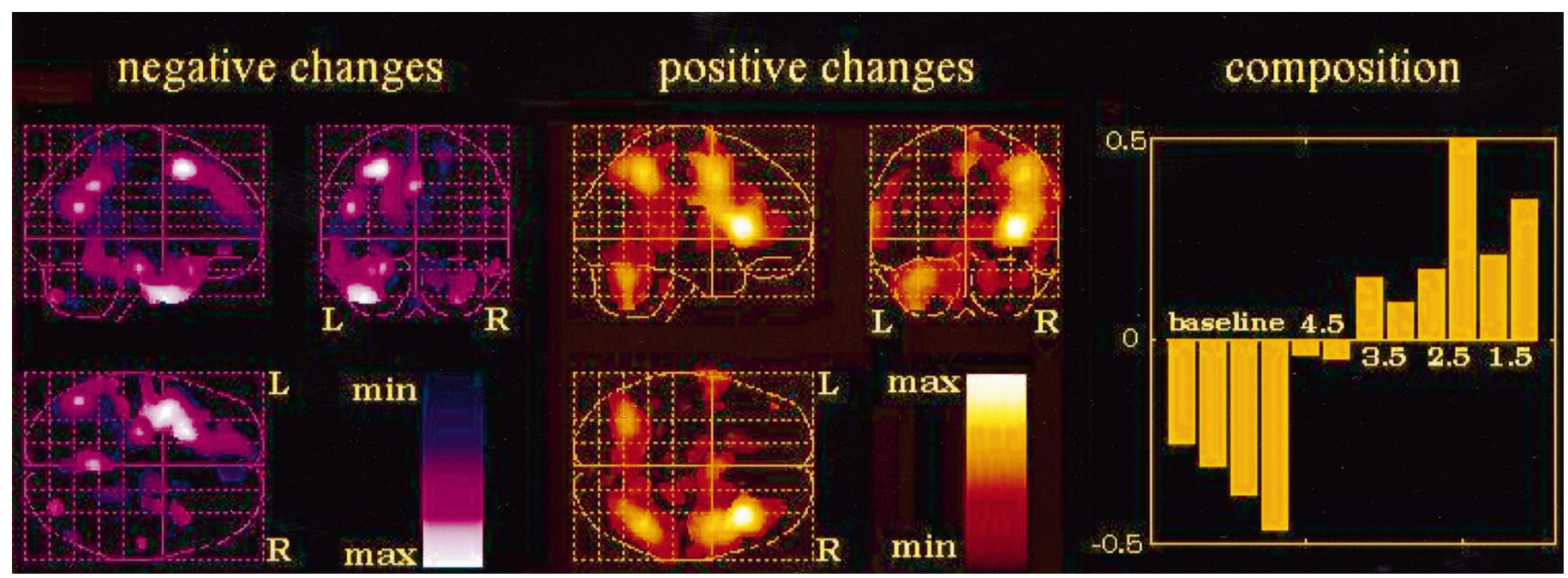

Figure 3. First eigenimage of the dataset (84 scans), explaining $56.4 \%$ of the variance. The corresponding activation/deactivation pattern is indicated by the left (negative changes) and middle (positive changes) panels. Composition of the eigenimage in terms of the scans corresponding to each experimental condition (four baseline scans; eight intensity discrimination tasks, two per $d^{\prime}$ value) is indicated in the right panel.

Although the excisions did not remove temporal cortex located as posteriorly as the region activated in the present study, these results confirm the role of the right temporal auditory regions in intensity discrimination. Third, in opposition to the modulation of the frontoparietal activity by intensity discriminability, no such relation was observed in the posterior temporal focus of activation. Figure 2 shows that mean activity in this region is mainly modulated by the presence or absence of the detection task and is roughly independent of the performance level in the detection tasks. This result suggests that this region is more specifically involved in sensory aspects of the detection, and in particular with operation of intensity change computation per se, which in principle should remain the same regardless of the actual physical differences detected, and therefore of the $d^{\prime}$ value for a given stimulus.

Interestingly, no modulation of activity could be observed in either left or right primary auditory cortices when the subjects switched from passive listening conditions to active detection of intensity differences. This observation is in agreement with recent data from a neuroimaging study of auditory attention by Zatorre et al. (1996). In this study, they scanned normal volunteers attending to spectral or spatial features of similar auditory stimulations, and observed activation of a right hemispheric frontoparietal network very similar to the one observed in the present study. Importantly however, activity in primary or secondary regions of the auditory cortex was not significantly different between the passive baseline condition and the different attending conditions. Another neuroimaging study confirms the absence of auditory cortex modulation by selective auditory attention (Platel et al., 1997). In this study, the authors scanned normal subjects who were selectively attending to rhythm, timbre, or pitch in the same sequences of notes. Although different cortical regions were found to be activated for the different attended features, no significant changes were observed in primary or secondary auditory cortices between the different selective attention tasks. These observations are consistent with a model of auditory perception according to which neural processing of acoustic features in primary auditory cortex would be to a large extent automatic and pre-attentive (Mondor et al., 1998).

These results, taken as a whole, suggest that the intensity discrimination activated two different sets of regions in the right cerebral hemisphere: (1) a general, nonspecific frontoparietal network involved in selective or sustained attention to sensory stimuli, which is consistently activated regardless of the particular sensory modality, and the activity of which is intimately related to the attentional resources required, and thus to intensity discriminability; and (2) a region of secondary auditory cortex in the right superior temporal gyrus, posterior to Heschl's gyrus, specifically involved in sensory aspects of detection and in particular in the computation of intensity differences, and the activity of which is largely independent of the physical differences to be computed and thus of the actual performance level.

\section{Parametric analysis versus eigenimages}

The parametric analysis we conducted on the dataset sought brain regions in which activity would be significantly correlated to performance level as measured by the $d^{\prime}$ value. Only one region in the right parietal lobe showed such a significant quasi-linear relation between $\mathrm{rCBF}$ and $d^{\prime}$ value. However, this analysis was very restraining in that it excluded a priori any region that would have a nonlinear $\mathrm{rCBF}-d^{\prime}$ relation. In contrast, the decomposition of the dataset into principal components, or "eigenimages," which introduces no a priori assumptions concerning the shape of the rCBF- $-d^{\prime}$ curve, showed that the activity in the whole frontoparietal network was indeed modulated by the performance level, although in a somewhat less linear way. This shows the power of the eigenimage approach, which simply consists of a mathematical simplification of the dataset.

The correlational analysis showed, nevertheless, the importance of using individually selected physical values corresponding to equivalent sensitivity levels for the activation tasks, rather than similar decibel values for all of the subjects. The significant correlation peak we obtained disappeared when physical levels (decibel values; see Table 1) were used instead of equivalent performance levels, suggesting that inter-individual variability is a confounding factor in this type of analysis. Indeed, if the same physical levels had been used in all the subjects for a given discriminability level, some subjects would have had a good performance and thus low activity in the attention-related frontoparietal network, whereas others would have had poorer per- 
formance and, accordingly, higher activity in the frontoparietal network. This would have disturbed the above results, obtained by either the correlational or the eigenimage approach. Careful assessment of individual levels of performance, by appropriate psychophysical techniques, is thus a crucial step in the realization of such studies.

The combination of psychoacoustics and functional neuroimaging thus appears successful in determining the cortical regions involved in perception and sustained attention to parameters of auditory stimulation such as intensity. In the considerable dataset acquired, we were able to tease apart activation of a nonspecific right frontoparietal attentional network from activation of a right posterior temporal region presumably specifically implicated in computation of intensity differences. In this analysis, the use of individual psychometric measures to take into account the considerable inter-individual variability in intensity discrimination and the use of powerful analysis techniques such as eigenimage analysis were of crucial importance.

\section{REFERENCES}

Belin P, Zilbovicius M, Crozier S, Thivard L, Fontaine A, Masure M-C, Samson Y (1998) Lateralization of speech and auditory temporal processing. J Cognit Neurosci, 10:536-540.

Bendriem B, Casey M, Dahlbom M, Trebossen R, Blohm K, Nutt R, Syrota A (1996) Evaluation of the ECAT EXACT HR+: a new positron camera with $2 \mathrm{D} / 3 \mathrm{D}$ acquisition capabilities and nearly isotropic spatial resolution. J Nucl Med, 37:170.

Binder JR, Rao SM, Hammeke TA, Yetkin FZ, Jesmanowicz A, Bandettini PA, Wong EC, Estkowski LD, Goldstein MD, Haughton VM, Hyde JS (1994) Functional magnetic resonance imaging of human auditory cortex. Ann Neurol 35:662-672.

Brugge JF, Reale RA (1985) Auditory cortex. In: Cerebral cortex, Vol 4 (Peters A, Jones EG, eds), pp 229-271. New York: Plenum.

Démonet JF, Chollet R, Ramsay S, Cardebat D, Nespoulos J, Wise R, Rascol A, Frackowiak RSJ (1992) The anatomy of phonological and semantic processing in normal subjects. Brain 115:1753-1768.

Démonet JF, Price C, Wise R, Frackowiak RSJ (1994) A PET study of cognitive strategies in normal subjects during language tasks: influence of phonetic ambiguity and sequence processing on phoneme monitoring. Brain 117:671-682.

Ehret G, Merzenich MM (1988) Complex sounds analysis (frequency resolution, filtering and spectral integration) by single units of the inferior colliculus of the cat. Brain Res Rev 13:139-163.

Fiez JA, Raichle ME, Miezin FM, Petersen SE, Tallal P, Katz WF (1995) PET studies of auditory and phonological processing: effects of stimulus characteristics and task demands. J Cognit Neurosci 7:357-375.

Fox PT, Mintun MA, Raichle ME, Herscovitch P (1984) A non-invasive approach to quantitative functional brain mapping with $\mathrm{H}_{2}{ }^{15} \mathrm{O}$ and positron emission tomography. J Cereb Blood Flow Metab 4:329-333.

Friston K, Ashburner J, Frith CD, Poline J-B, Heather JD, Frackowiak RSJ (1995a) Spatial registration and normalization of images. Hum Brain Mapp 2:165-189.

Friston K, Holmes AP, Worsley KJ, Poline J-B, Frith CD, Frackowiak RSJ (1995b) Statistical parametric mapping in functional imaging: a general linear approach. Hum Brain Mapp 2:189-210.

Giard MH, Lavikainen J, Reinikainen K, Perrin F, Bertrand O, Pernier J, Näätänen R (1995) Separate representations of stimulus frequency, intensity and duration in auditory sensory memory: an event-related potential and dipole model analysis. J Cognit Neurosci 7:113-143.

Gitelman DR, Alpert NM, Kosslyn S, Daffner K, Scinto L, Thompson W,
Mesulam M-M (1996) Functional imaging of human right hemispheric activation for exploratory movements. Ann Neurol 39:174-179.

Green DM, Swets JA (1974) Signal detection theory and psychophysics. New York: Krieger.

Heil P, Irvine DRF (1998) The posterior field P of cat auditory cortex: coding of envelope transients. Cereb Cortex 8:125-141.

Joanette Y, Goulet P, Hannequin D (1994) Troubles de la communication verbale chez les droitiers cérébro-lésés droits. In: Neuropsychologie humaine (Seron X, and Jeannerod M, eds), pp 342-344. Liège: Mardaga.

Johnsrude IS, Zatorre RJ, Milner BA, Evans AC (1997) Lefthemisphere specialization for the processing of acoustic transients. NeuroReport 8:1761-1765.

Knopman DS, Rubens AB, Klassen AC, Meyer MW (1982) Regional cerebral blood flow correlates of auditory processing. Arch Neurol 39:487-493.

Levitt H (1971) Transformed up-down methods in psychoacoustics. J Acoust Soc Am 49:467-477.

Macmillan N, Creelman D (1990) Detection theory: a user's guide. Cambridge, UK: Cambridge UP.

Mesulam M-M (1981) A cortical network for directed attention and unilateral neglect. Ann Neurol 10:309-325.

Milner B (1962) Laterality effects in audition. In: Interhemispheric relations and cerebral dominance (Mountcastle VB, ed), pp 177-195. Baltimore: John Hopkins Press.

Mondor TA, Zatorre RJ, Terrio NA (1998) Constraints on the selection of auditory information. J Exp Psychol Hum Percept Perform, in press.

Monrad-Krohn GH (1963) The third element of speech: prosody and its disorders. In: Problems of dynamic neurology (Halpern L, ed), pp 101-117. Jerusalem: Hebrew UP.

Näätänen R (1990) The role of attention in auditory information processing as revealed by event-related potentials and other brain measures of cognitive function. Behav Brain Res 13:201-288.

O'Leary DS, Andreasen NC, Hurtig R, Flashman L, Torres I, Hichwa R (1995) A PET study of auditory and visual attention. Soc Neurosci Abstr 21:1988.

Pardo JV, Fox PT, Raichle ME (1991) Localization of a human system for sustained attention by positron emission tomography. Nature 349:61-64.

Paus T (1996) Location and function of the human frontal eye-field: a selective review. Neuropsychologia 34:475-483.

Paus T, Zatorre RJ, Hofle N, Caramanos Z, Gotman J, Petrides M, Evans AC (1997) Time-related changes in neural systems underlying attention and arousal during the performance of an auditory vigilance task. J Cognit Neurosci 9:392-408.

Platel H, Price C, Baron JC, Wise R, Lambert J, Frackowiak RSJ, Lechevalier B, Eustache F (1997) The structural components of music perception. A functional anatomical study. Brain 120:229-243.

Talairach J, Tournoux P (1988) Co-Planar stereotaxic atlas of the human brain. New York: Thieme.

Wessinger C, Tian B, VanMeter JW, Platenberg RC, Pekar J, Rauscheker JP (1997) Hierarchical processing within human auditory cortex examined with fMRI. Soc Neurosci Abstr 23:2073.

Wilkins AJ, Shallice T, McCarthy R (1987) Frontal lesions and sustained attention. Neuropsychologia 25:359-365.

Woods DL, Knight RT (1986) Electrophysiologic evidence of increased distractibility after dorsolateral prefrontal lesions. Neurology 36:212-216.

Zatorre RJ, Evans AC, Meyer E, Gjedde A (1992) Lateralisation of phonetic and pitch discrimination in speech processing. Science 256:846-849.

Zatorre RJ, Meyer E, Gjedde A, Evans AC (1996) PET studies of phonetic processing of speech: review, replication and reanalysis. Cereb Cortex 6:21-30. 